\title{
Two-pion interferometry for the granular sources in ultrarelativistic heavy ion collisions
}

\author{
Wei-Ning Zhang ${ }^{1,2 *}$, Hong-Jie Yin ${ }^{1}$, and Yan-Yu Ren ${ }^{2}$ \\ ${ }^{1}$ School of Physics and Optoelectronic Technology, \\ Dalian University of Technology, \\ Dalian, Liaoning 116024, China \\ ${ }^{2}$ Department of Physics, Harbin Institute of Technology, \\ Harbin, Heilongjiang 150006, China
}

\begin{abstract}
We investigate the two-pion interferometry in ultrarelativistic heavy ion collisions in the granular source model of quark-gluon plasma droplets. The pion transverse momentum spectra and HBT radii of the granular sources agree well with the experimental data of the $\sqrt{s_{N N}}=200 \mathrm{GeV} \mathrm{Au}-\mathrm{Au}$ and $\sqrt{s_{N N}}=2.76 \mathrm{TeV} \mathrm{Pb}-\mathrm{Pb}$ most central collisions. In the granular source model the larger initial system breakup time may lead to the larger HBT radii $R_{\text {out }}, R_{\text {side }}$, and $R_{\text {long }}$. However, the large droplet transverse expansion and limited average relative emitting time of particles in the granular source lead to small ratios of the transverse HBT radii $R_{\text {out }} / R_{\text {side }}$.

PACS numbers: 25.75.-q, 25.75.Gz
\end{abstract}

Hanbury-Brown-Twiss (HBT) interferometry is a useful tool to probe the space-time geometry of the particleemitting sources in high energy heavy ion collisions [14]. The experimental results of the HBT measurements for the $\mathrm{Au}-\mathrm{Au}$ collisions at the high energies of the Relativistic Heavy Ion Collider (RHIC) indicate that it is hard to describe the source space-time by a simple evolution model [5 8]. HBT interferometry data provide strong constraints for the models of source space-time. Recently, the HBT measurement for the $\sqrt{s_{N N}}=2.76$ $\mathrm{TeV} \mathrm{Pb}-\mathrm{Pb}$ most central collisions at the Large Hadron Collider (LHC) is performed [9]. A consistent explanation to the HBT data of the LHC and RHIC experiments is required naturally for the source models, which will be helpful to understand the initial condition, source evolution, and particle freeze-out in ultrarelativistic heavy ion collisions.

In Refs. [10, 11], the granular source model of quarkgluon plasma (QGP) droplets [12] is developed to explain the RHIC HBT data [7, 8]. In this work we investigate the two-pion HBT interferometry in ultrarelativistic heavy ion collisions in the granular source model of QGP droplets. Our results indicate that the granular source for the LHC Pb-Pb collisions may have the same initial droplet temperature and velocity formula as those for the RHIC Au-Au collisions, but a larger initial system breakup time. The consistent granular source model reproduces the pion transverse momentum spectra and HBT radii in the most central collisions of the RHIC [8, 13, 14] and LHC [9, 15] experiments.

In ultrarelativistic heavy ion collisions, the system at central rapidity may reach a local equilibrium at a very short time $\tau_{0}$, then fast expand in the beam direction (z-axis). Because of the initial fluctuation, the local-

\footnotetext{
*wnzhang@dlut.edu.cn
}

equilibrium system is not uniform in space [16, 17]. It may form many tubes along the beam direction during the fast longitudinal expansion, and finally fragment into many droplets (see Fig.1 of Ref. [10]) due to the "sausage" instability and surface tension [10]. On the other hand, the rapidly increased bulk viscosity in the QGP near the phase transition may also leads to the system breakup [18].

We assume that the system fragments and forms a granular source of many QGP droplets at a time $t_{0}\left(>\tau_{0}\right)$. On the basis of the Bjorken hypothesis [19], the longitudinal velocity and rapidity of the droplets at $t_{0}$ are

$$
v_{d z}=z_{0} / t_{0}, \quad \eta_{0}=\frac{1}{2} \log \frac{t_{0}+z_{0}}{t_{0}-z_{0}},
$$

and the transverse velocity of the droplets may be expressed as [11, 20]

$$
v_{d \perp}=a_{T}\left(\frac{\rho_{0}}{\mathcal{R}_{\perp 0}}\right)^{b_{T}} \sqrt{1-v_{d z}^{2}},
$$

where $z_{0}$ and $\rho_{0}$ are the longitudinal and transverse coordinates of the droplet at the breakup time $t_{0}, \mathcal{R}_{\perp 0}$ is the maximum transverse radius of the system at $t_{0}$. In Eq. (2), the quantities $a_{T}$ and $b_{T}$ are the magnitude and power parameters of the transverse velocity which will be determined by the data of particle transverse momentum spectra.

In our model calculations, the initial droplet radius in droplet local frame satisfies a Gaussian distribution with standard deviation $\sigma_{d}$, and the initial droplet centers are assumed distributing within a cylinder along the beam direction by [10, 11]

$$
\frac{d N}{2 \pi \rho_{0} d \rho_{0}} \propto\left[1-\exp \left(-\rho_{0}^{2} / \Delta \mathcal{R}_{\perp}^{2}\right)\right] \theta\left(\mathcal{R}_{\perp}-\rho_{0}\right),
$$

where $\mathcal{R}_{\perp}$ and $\Delta \mathcal{R}_{\perp}$ are the initial transverse radius and shell parameter of the granular source [10, 11]. Because of 
the longitudinal boost-invariant in ultrarelativistic heavy ion collisions, we may obtain the initial coordinate $z_{0}$ of the droplet by the longitudinal distribution

$$
\frac{d N}{d z_{0}}=\frac{d N}{d \eta_{0}} \frac{d \eta_{0}}{d z_{0}} \propto 1 \cdot \frac{t_{0}}{t_{0}^{2}-z_{0}^{2}}, \quad\left|z_{0}\right|<\sqrt{t_{0}^{2}-\tau_{0}^{2}} .
$$

The evolution of the granular source after $t_{0}$ is the superposition of all the evolutions of the individual droplets, each of them is described by relativistic hydrodynamics with an initial local energy density $\epsilon_{0}$ and the equation of state (EOS) of the entropy density with a cross over between the QGP and hadronic gas [21 23]. The values of the EOS parameters used in the calculations are taken as the same as in Ref. 11]. In order to include the pions emitted directly at hadronization and decayed from resonances later, we let the pions freeze-out within a wide temperature region with the probability [11]

$$
\begin{aligned}
\frac{d P_{f}}{d T} & \propto f_{\mathrm{dir}} e^{-\left(T_{c}-T\right) / \Delta T_{\mathrm{dir}}}+\left(1-f_{\mathrm{dir}}\right) \\
& \times e^{-\left(T_{c}-T\right) / \Delta T_{\mathrm{dec}}}, \quad\left(T_{c}>T>80 \mathrm{MeV}\right),
\end{aligned}
$$

where $T_{c}$ is the transition temperature, $f_{\text {dir }}$ is a fraction parameter for the direct emission, $\Delta T_{\mathrm{dir}}$ and $\Delta T_{\mathrm{dec}}$ describe the widths of temperature for the direct and decayed pion emissions. They are taken to be $T_{c}=170$ $\mathrm{MeV}, f_{\text {dir }}=0.85, T_{\text {dir }}=10 \mathrm{MeV}$, and $T_{\text {dec }}=90 \mathrm{MeV}$ [11.

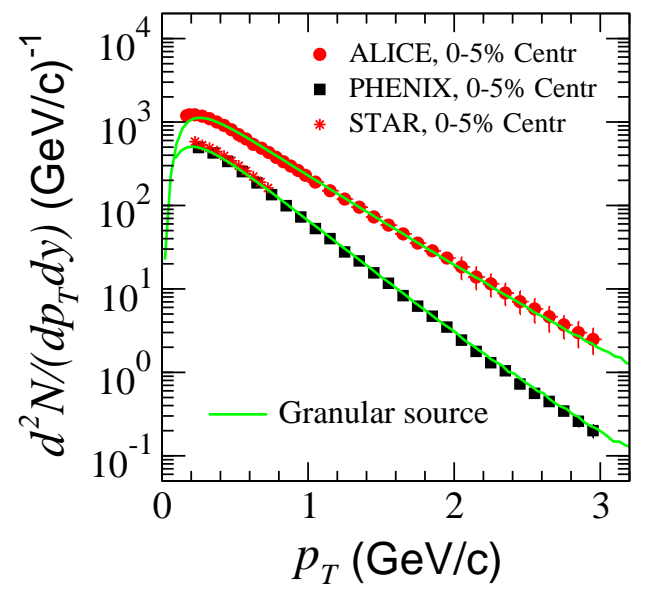

FIG. 1: (Color online) The pion transverse momentum distribution of the granular source and the experimental data of $\sqrt{s_{N N}}=200 \mathrm{GeV} \mathrm{Au-Au} \mathrm{(PHENIX} \mathrm{13]} \mathrm{and} \mathrm{STAR} \mathrm{14])} \mathrm{and}$ $\sqrt{s_{N N}}=2.76 \mathrm{TeV} \mathrm{Pb}-\mathrm{Pb}$ (ALICE 15]) most central collisions.

In Fig. 1, we show the pion transverse momentum spectra calculated with the granular source model and the experimental data of $\sqrt{s_{N N}}=200 \mathrm{GeV} \mathrm{Au}-\mathrm{Au}$ [13, 14] and $\sqrt{s_{N N}}=2.76 \mathrm{TeV} \mathrm{Pb}-\mathrm{Pb}$ [15] most central collisions. Assuming that the systems fragment when the local energy density decreases at a certain value, we take the initial droplet temperature $T_{0}=200 \mathrm{MeV}$ [11] in the calculations. The corresponding initial energy density of the droplets, $\epsilon_{0}$, is $2.24 \mathrm{GeV} / \mathrm{fm}^{3}$. The standard deviation $\sigma_{d}$ for the droplet radius distribution is taken to be $2.5 \mathrm{fm}$. For the granular source in the RHIC collisions, $\tau_{0}$ and the breakup time $t_{0}$ are taken to be 0.8 and $4.3 \mathrm{fm} / \mathrm{c}$, and the source parameters $\mathcal{R}_{\perp}$ and $\Delta \mathcal{R}_{\perp}$ are taken to be 6.5 and $3.4 \mathrm{fm}$, respectively. For the granular source in the LHC collisions, these parameters are taken to be $\tau_{0}=0.4 \mathrm{fm} / \mathrm{c}, t_{0}=8.0 \mathrm{fm} / \mathrm{c}, \mathcal{R}_{\perp}=7.8$ $\mathrm{fm}$, and $\Delta \mathcal{R}_{\perp}=5.5 \mathrm{fm}$. By comparing the transverse momentum spectra of the granular sources with the data of $\sqrt{s_{N N}}=200 \mathrm{GeV} \mathrm{Au}-\mathrm{Au}[13,14]$ and $\sqrt{s_{N N}}=2.76$ $\mathrm{TeV} \mathrm{Pb}-\mathrm{Pb}$ [15] most central collisions, we fix the parameters of the droplet transverse velocity $a_{T}=0.65$ and $b_{T}=1.40$ the same for both the two energy sources in Eq. (2), where $\mathcal{R}_{\perp 0}$ is taken to be $8.0 \mathrm{fm}$ in the calculations.
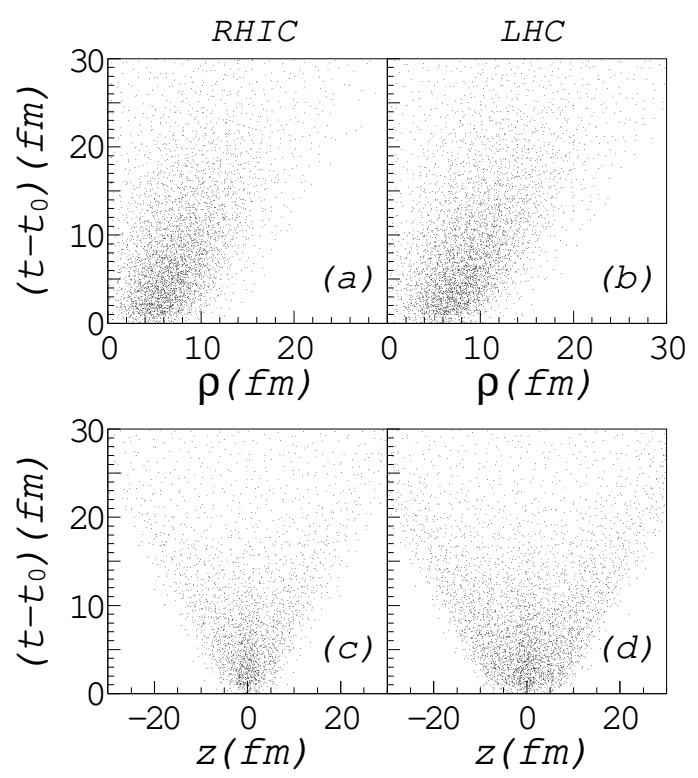

FIG. 2: The space-time distribution of pion-emitting points in the granular source model.

Figure 2] shows the space-time distributions of the pionemitting points in the granular source model. Because of the higher collision energy, the granular source for the LHC collisions forms (system energy density decreases to a certain value) at the larger $t_{0}$, and hence has a wider $z_{0}$ distribution (see Eq. (44) and larger $\mathcal{R}_{\perp}$ value (wider $\rho_{0}$ distribution, see Eq. (3)). These lead to the wider distributions of the longitudinal and transverse source points for the LHC granular source than those for the RHIC granular source.

In Fig. 3, we show the HBT radii $R_{\text {out }}, R_{\text {side }}$ and $R_{\text {long }}$ in the "out", "side", and "long" directions [24, 25], and the chaotic parameter $\lambda$ of the granular sources as functions of the transverse pion pair momentum, $k_{T}=$ $\left|\mathbf{p}_{1 T}+\mathbf{p}_{2 T}\right| / 2$. They are obtained by fitting the two- 


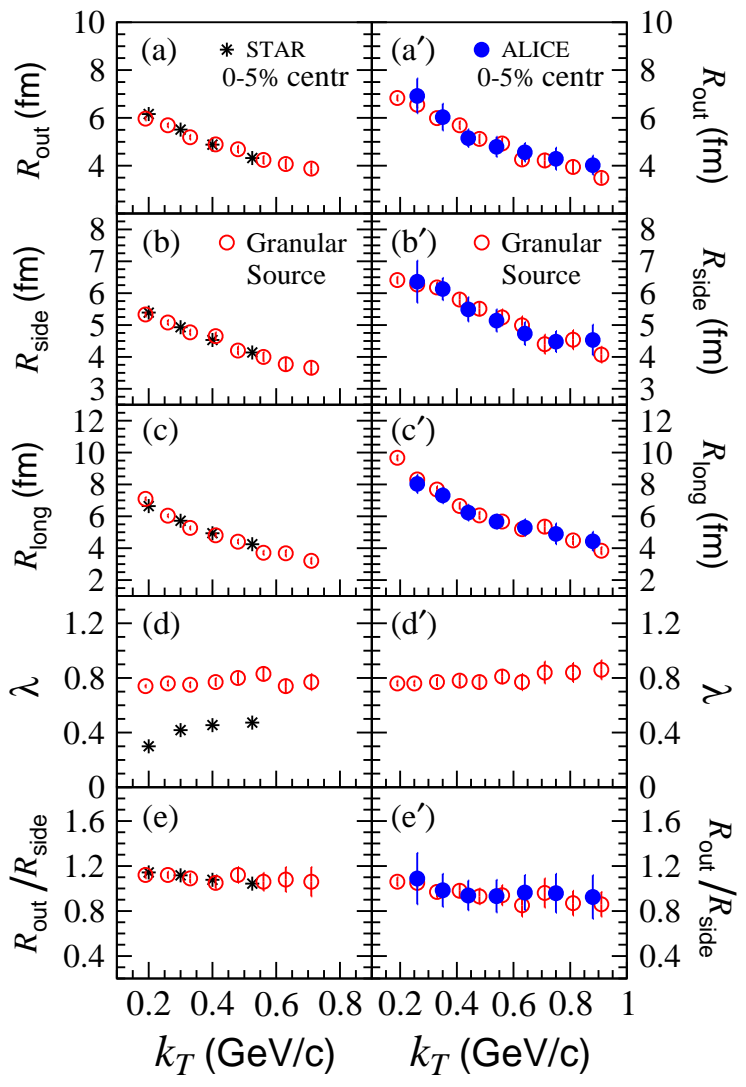

FIG. 3: (Color online) (a) The two-pion interferometry results of the granular sources and the experimental HBT data for the most central collisions of $\sqrt{s_{N N}}=200 \mathrm{GeV} \mathrm{Au}-\mathrm{Au}$ [8] and $\sqrt{s_{N N}}=2.76 \mathrm{TeV} \mathrm{Pb}-\mathrm{Pb}[9]$.

pion correlation functions in different $k_{T}$ regions with the formula

$C\left(q_{\text {out }}, q_{\text {side }}, q_{\text {long }}\right)=1+\lambda e^{-q_{\text {out }}^{2} R_{\text {out }}^{2}-q_{\text {side }}^{2} R_{\text {side }}^{2}-q_{\text {long }}^{2} R_{\text {long }}^{2}}$,

in the longitudinal comoving system (LCMS) [4]. The HBT data of the most central collisions of $\sqrt{s_{N N}}=200$ $\mathrm{GeV} \mathrm{Au-Au} \mathrm{(STAR} \mathrm{[8])} \mathrm{and} \sqrt{s_{N N}}=2.76 \mathrm{TeV} \mathrm{Pb}-\mathrm{Pb}$ (ALICE [9]) are also shown. In our calculations, we use the same cuts as in the experimental analyses [8, 9], that is the pion rapidity is limited with $|y|<0.5$ for the granular source for the RHIC collisions and the pion pseudorapidity satisfies $|\eta|<0.8$ for the granular source for the LHC collisions, respectively. It can be seen that the HBT radii of the granular sources agree well with the experimental data. The wider distributions of the transverse and longitudinal source points for the LHC granular source (see Fig. 2) lead to the larger transverse and longitudinal HBT radii than those for the RHIC granular source. In experimental HBT measurements, many effects, such as the Coulomb interaction between the final particles, particle missing-identification, source coherence, etc., can influence the results of the $\lambda[1-4]$. Because we do not consider these effects in our model, our $\lambda$ results are larger.
The ratio of the transverse $\mathrm{HBT}$ radii $R_{\text {out }} / R_{\text {side }}$ is related to the average relative emitting time of the two pions and the velocity of the source transverse expansion, $\overline{\Delta t}=\left\langle\left|t_{1}-t_{2}\right|\right\rangle$ and $v_{\mathrm{ST}}, 2$, 4]. Although the larger breakup time $t_{0}$ for the LHC source leads to a larger particle-emitting time, the limited value of $\overline{\Delta t}$ for the granular sources [10 12, 26] and the large droplet transverse velocity lead to the small $R_{\text {out }} / R_{\text {side }}$ results (see Fig. 3 (e) and (e')). In the upper panel of Fig. 4, we show the average pion-emitting time $\bar{t}$ and the average relative pion-emitting time $\overline{\Delta t}$ for the RHIC and LHC granular sources. It can be seen that the average pionemitting time for the LHC source is much larger than that for the RHIC source. However, there is only small difference between the average relative pion-emitting times for the LHC and RHIC sources. In the lower panel of Fig. 4, we show the average source transverse and longitudinal velocities as functions of the transverse pion pair momentum $k_{T}$. The transverse velocity $\bar{v}_{\mathrm{ST}}$ increases with $k_{T}$, and the longitudinal velocity $\bar{v}_{\mathrm{SL}}$ decreases with $k_{T}$. For the collisions at the LHC energy the granular source has larger transverse and longitudinal velocities.

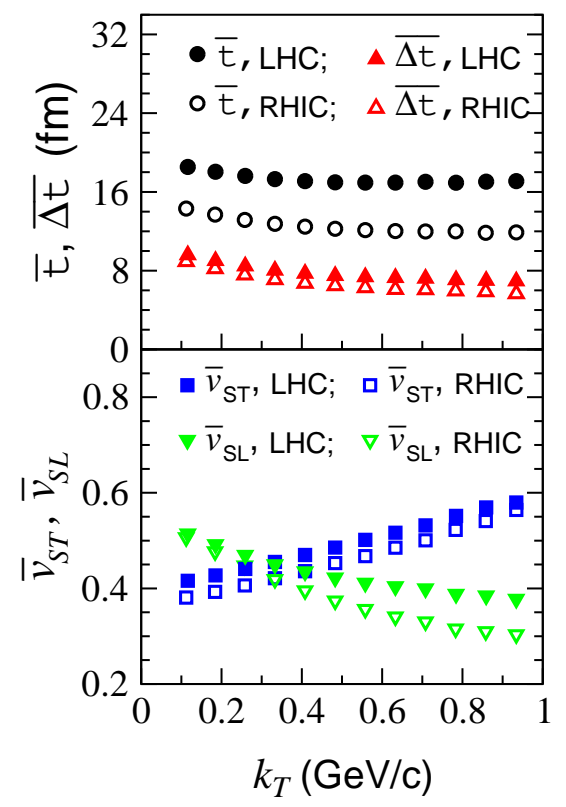

FIG. 4: (Color online) Upper panel: the average pionemitting time and relative pion-emitting time for the granular sources. Lower panel: the average source transverse and longitudinal velocities for the granular sources.

In summary, we investigate the two-pion HBT interferometry in ultrarelativistic heavy ion collisions in the granular source model of QGP droplets. The pion transverse momentum spectra and HBT radii of the granular sources agree well with the RHIC $\sqrt{s_{N N}}=200 \mathrm{GeV} \mathrm{Au}$ $\mathrm{Au}$ and $\mathrm{LHC} \sqrt{s_{N N}}=2.76 \mathrm{TeV} \mathrm{Pb}-\mathrm{Pb}$ most central collisions. Our results indicate that the granular source for the collisions at the LHC energy may have the same ini- 
tial droplet temperature and velocity formula as those for the collisions at the RHIC energy, but a larger initial system breakup time. The larger breakup time may lead to the larger longitudinal and transverse distributions of the source points, and hence lead to the larger longitudinal and transverse HBT radii. However, the average relative emitting time of the two pions is limited in the granular source model. The limited relative pion-emitting time and larger droplet transverse expansion lead to the smaller ratio of the transverse HBT radii, $R_{\text {out }} / R_{\text {side. }}$ In our granular source model, there is correlations between the space-time coordinates and source velocities. The data of the particle transverse momentum spectra and HBT radii for the collisions at the RHIC and LHC en- ergies give strong constraints for the model parameters. The consistent explain of the granular source model to these experimental data will be helpful to understand the formation and evolution of the pion-emitting sources in ultrarelativistic heavy ion collisions.

\section{Acknowledgments}

This research was supported by the National Natural Science Foundation of China under Contract No. 11075027.
[1] C. Y. Wong, Introduction to High-Energy Heavy-Ion Collisions (World Scientific, Singapore, 1994), Chap. 17.

[2] U. A. Wiedemann and U. Heinz, Phys. Rept. 319, 145 (1999).

[3] R. M. Weiner, Phys. Rept. 327, 249 (2000).

[4] M. A. Lisa, S. Pratt, R. Soltz, U. Wiedemann, Ann. Rev. Nucl. Part. Sci. 55, 357 (2005); nucl-ex/0505014

[5] C. Adler et al. (STAR Collaboration), Phys. Rev. Lett. 87, 082301 (2001).

[6] K. Adcox et al. (PHENIX Collaboration), Phys. Rev. Lett. 88, 192302 (2002).

[7] S. S. Adler et al. (PHENIX Collaboration), Phys. Rev. Lett. 93, 152302 (2004).

[8] J. Adams et al. (STAR Collaboration), Phys. Rev. C 71, 044906 (2005).

[9] K. Aamodt et al. (ALICE Collaboration), Phys. Lett. B 696328 (2011).

[10] W. N. Zhang, Y. Y. Ren, and C. Y. Wong, Phys. Rev. C 74, 024908 (2006).

[11] W. N. Zhang, Z. T. Yang, and Y. Y. Ren, Phys. Rev. C 80, 044908 (2009).

[12] W. N. Zhang, M. J. Efaaf, and C. Y. Wong, Phys. Rev. C 70, 024903 (2004).

[13] S. S. Adler et al. (PHENIX Collaboration), Phys. Rev. C 69, 034909 (2004).
[14] J. Adams et al. (STAR Collaboration), Phys. Rev. Lett. 92, 112301 (2004).

[15] M. Floris on behalf of ALICE Collaboration, plenary talk at Quark Matter 2011, May 26, 2011, Annecy, France.

[16] H. J. Drescher, F. M. Liu, S. Ostapchenko, T. Pierog, and K. Werner, Phys. Rev. C 65, 054902 (2002).

[17] O. Socolowski Jr., E. Grassi, Y. Hama, and T. Kodama, Phys. Rev. Lett. 93, 182301 (2004).

[18] G. Torrieri, B. Tomášik, I. Mishustin, Phys. Rev. C 77, 034903 (2008).

[19] J. D. Bjorken, Phys. Rev. D 27, 140 (1983).

[20] G. Baym, B. L. Friman, J. P. Blazot, M. Soyeur, and W. Czyz, Nucl. Phys. A407, 541 (1983).

[21] J. P. Blaizot J. Y. Ollitrault, Phys. Rev. D 36, 916 (1987).

[22] D. H. Rischke and M. Gyulassy, Nucl. Phys. A608, 479 (1996).

[23] E. Laermann, Nucl. Phys. A610, 1 (1996).

[24] G. Bertsch, M. Gong, M. Tohyama, Phys. Rev. C 37, 1896 (1988); G. Bertsch, Nucl. Phys. A 498, 173c (1989).

[25] S. Pratt, T. Csörgő and J. Zimányi, Phys. Rev. C 42, 2646 (1990).

[26] Wei-Ning Zhang and Cheuk-Yin Wong, Int. J. Mod. Phys. E 16, 3262 (2007) 